\title{
A Smart and Accurate Interface for Resistive Sensors
}

\author{
Xiujun Li and Gerard C. M. Meijer, Senior Member, IEEE
}

\begin{abstract}
This paper presents a smart and accurate interface for resistive sensors based on the use of a relaxation oscillator. To obtain high accuracy, good long-term stability and a reduction of the effects of interference and parasitic elements, some classical and new measurement techniques have been applied in the novel sensor interface. Moreover, all multiplicative and additive errors, and the common-mode effect of the interface are eliminated, using a multiple-signal calibration technique. A prototype has been built and was tested. Experimental results show that the interface is able to measure a resistance of 0 to $400 \Omega$, with a resolution of $7 \mathrm{~m} \Omega$ and an accuracy of $11 \mathrm{~m} \Omega$. The measurement time is about $100 \mathrm{~ms}$.
\end{abstract}

Index Terms-Auto-calibration, interface, relaxation oscillator, resistive sensor.

\section{INTRODUCTION}

$\mathbf{R}$ ESISTIVE sensors, such as platinum resistors and thermistors, are widely applied for temperature measurements [1], [2]. To perform an accurate temperature measurement using these sensors, one needs a very accurate resistive measurement. For instance, when an accuracy of $0.04{ }^{\circ} \mathrm{C}$ for the temperature measurement is required, a platinum resistor $(100 \Omega)$ should be measured with an accuracy of $16 \mathrm{~m} \Omega$. Some classical and some new measurement techniques, for instance, the chopping technique, four-wire measurement, three-signal calibration and the ac excitation, can be used to get rid of many nonidealities in the resistance measurement [3]. However, the use of an ac square-wave excitation signal in combination with the switched-capacitor sampling technique can cause a significant common-mode effect via the parasitic capacitors.

In this paper, we propose using a multiple-signal calibration technique to reduce the common-mode effect in the resistivesensor interface. The proposed interface is very suitable for implementation in low-cost CMOS technology.

\section{MEASUREMENT CONCEPT}

Many classical and new measurement techniques can be applied to obtain a high-performance sensor interface [3], [4]. For instance, the four-wire measurement technique is used to reduce the effect of series impedances caused by the wires and cables used to connect the resistive sensor to the interface circuit. The ac square-wave excitation signal for the sensing element is used to reduce the effect of the low-frequency drift and the influence of parasitic Seebeck voltages. However, the ac square-wave excitation signal can also be used to implement

\footnotetext{
Manuscript received May 4, 2000; revised September 30, 2001. This work was supported by the Dutch Technology Foundation (STW), The Netherlands, under the Project DEL 4540.

The authors are with the Faculty of Information Technology and Systems, Delft University of Technology, Delft, The Netherlands (e-mail: X.Li@ITS.tudelft.nl).

Publisher Item Identifier S 0018-9456(01)10969-1.
}

an advanced chopping technique [4]. The use of the chopping technique can significantly reduce the effect of offset, $1 / \mathrm{f}$ noise, and low-frequency interference. This enables the use of low-cost CMOS technology for accurate measurement systems. However, the use of the ac square-wave excitation signal, the chopping technique and the switched-capacitor sampling technique will cause a significant common-mode effect via parasitic capacitors. Even when the advanced three-signal auto-calibration technique is used [5], this common-mode effect is not eliminated completely. To reduce the common-mode effect, we propose using a multiple-signal calibration technique, which is described in the following.

As [5], [6] indicates, the three-signal auto-calibration technique is applied to eliminate the multiplicative and offset parameters of the interface. In order to apply this technique, we must measure not only the sensor signal (in our case, resistance) $E_{x}$, but also a reference signal $E_{r e f}$ and an offset of the total interface in a linear system with output signals $M_{i}$

$$
M_{i}=k E_{i}+M_{o f f} .
$$

After the three-signal auto-calibration technique has been applied, the final measuring result is the ratio

$$
M=\frac{M_{x}-M_{o f f}}{M_{r e f}-M_{o f f}}=\frac{E_{x}}{E_{r e f}} .
$$

In this ratio, the influences of the unknown offset $M_{o f f}$ and the unknown gain $k$ of the measurement system are eliminated.

According to the experimental results, the common-mode effect affects the offset, but not the gain $k$ in the measurement result $M_{i}$. The common-mode voltages are different for the three phases of the measurement. Therefore, instead of a single offset contribution there are two different contributions: $M_{o f f x}$ and $M_{o f f r e f}$ for two measurements $M_{x}$ and $M_{r e f}$, respectively. A solution for this problem is that two offsets, $M_{o f f x}$ and $M_{o f f r e f}$, are measured for both measurements of $M_{x}$ and $M_{\text {ref }}$, respectively. Then, with respect to the common-mode effect, eq. (2) can be rewritten as

$$
M=\frac{M_{x}-M_{o f f x}}{M_{r e f}-M_{o f f r e f}}=\frac{E_{x}}{E_{r e f}} .
$$

\section{DESIGN OF THE INTERFACE}

Fig. 1(a) shows a simplified schematic diagram of the interface for resistive sensors. Fig. 1(b) shows some signals of the interface when one of $M_{o f f x}, M_{o f f r e f}, M_{x}$, and $M_{\text {ref }}$ is measured. The interface mainly consists of a relaxation oscillator, a multiplexer and a logic control circuit. In this figure, $R_{x}$ denotes the resistive sensor, for instance, a Pt100. The resistor $R_{r e f}$ is a well-known reference resistor with a low temperature coefficient and high stability. 


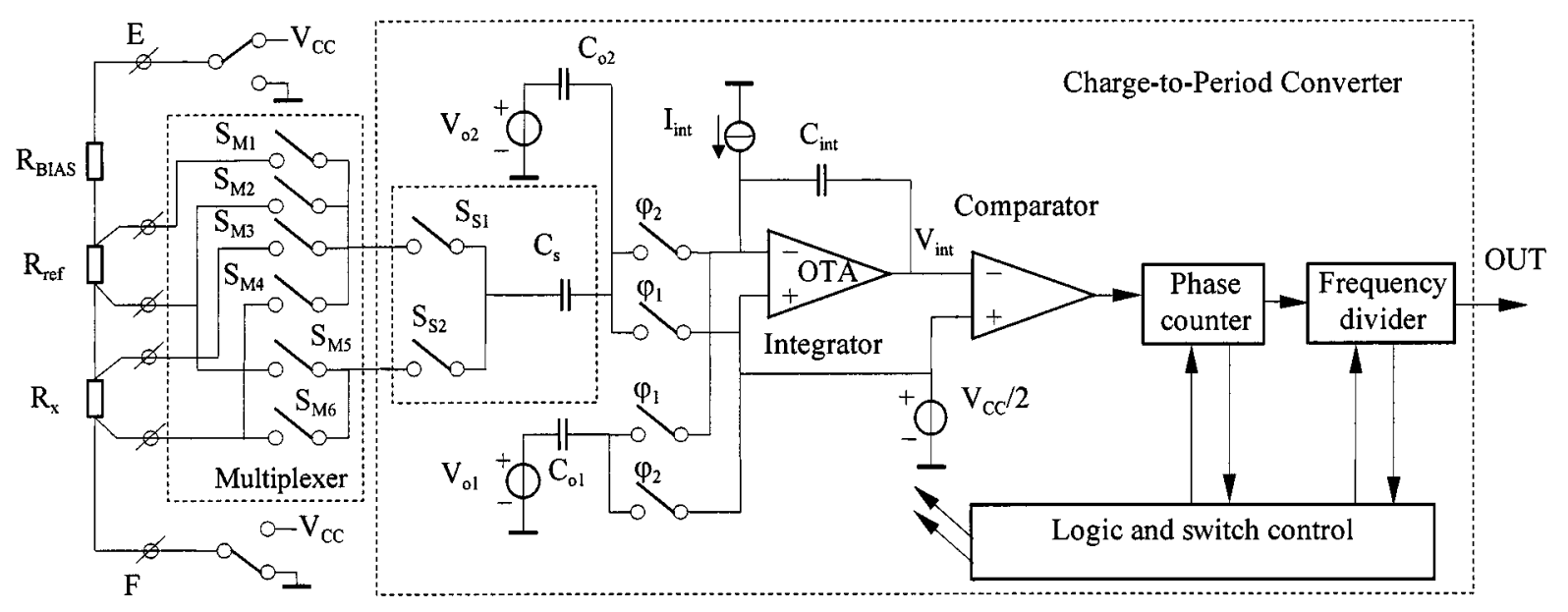

(a)

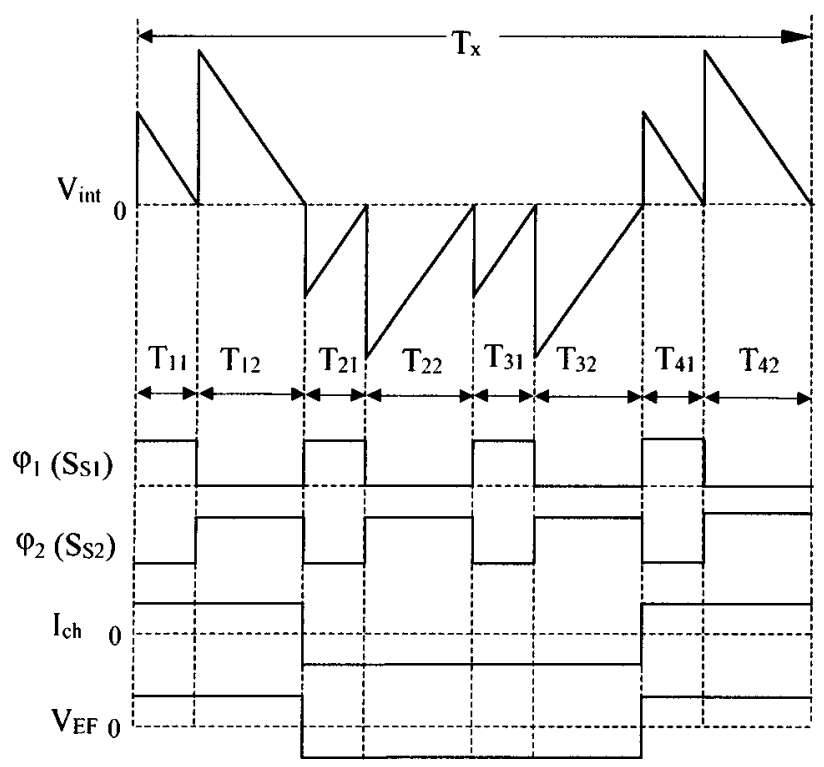

(b)

Fig. 1. (a) Schematic diagram and (b) some signals of the interface.

The relaxation oscillator and the switched-capacitor sampler linearly convert the voltage values to period-modulated signals. The multiplexer selects the signal to be measured. Table I lists the situations of switches in the multiplexer when different signals are measured. The control logic will control the situation of all switches. The interface outputs a microcontroller-compatible signal.

In this interface, to apply the chopping technique, two square-wave signals with amplitude of $V_{C C}$ are generated at points $\mathrm{E}$ and $\mathrm{F}$ to drive the resistor path. These two signals have the same amplitude and a phase shift of $180^{\circ}$. Due to this square-wave driving signal, a large CM ac signal exists. The magnitude of this CM signal is different for $R_{r e f}$ and $R_{x}$, respectively (see Fig. 2). This causes the problem of unequal offset voltages for both measurements.

\section{NONIDEALITIES}

As described in Section II, many nonidealities of the interface are eliminated by means of the multiple-signal calibration
TABLE I

SituATions of SWITCHES IN THE MULTIPLEXeR

\begin{tabular}{c|c|c|c|c|c|c}
\hline Meas. & \multicolumn{6}{|c}{ Situation of MUX } \\
\cline { 2 - 7 } Signal & $\mathrm{S}_{\mathrm{M} 1}$ & $\mathrm{~S}_{\mathrm{M} 2}$ & $\mathrm{~S}_{\mathrm{M} 3}$ & $\mathrm{~S}_{\mathrm{M} 4}$ & $\mathrm{~S}_{\mathrm{M} 5}$ & $\mathrm{~S}_{\mathrm{M} 6}$ \\
\hline$M_{\text {offref }}$ & 0 & 1 & 0 & 0 & 1 & 0 \\
$M_{\text {ref }}$ & 1 & 0 & 0 & 0 & 1 & 0 \\
$M_{\text {offx }}$ & 0 & 0 & 0 & 1 & 0 & 1 \\
$M_{\mathrm{x}}$ & 0 & 0 & 1 & 0 & 0 & 1 \\
\hline
\end{tabular}

technique. However, some effects cannot be eliminated by this technique and should be taken into account during the design of this interface.

\section{A. High-Frequency Pole}

The resistors $R_{x}, R_{r e f}$, and $R_{B I A S}$ and the capacitances associated with these resistors form an RC circuit. For instance, 


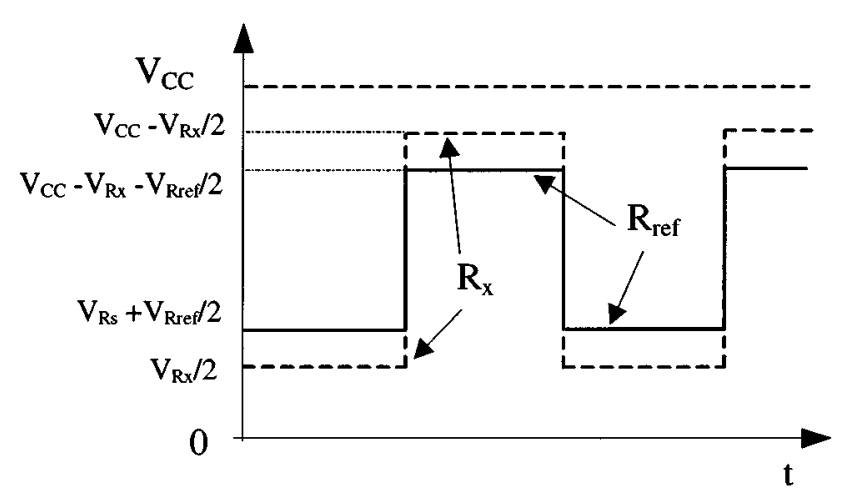

Fig. 2. Averaged common mode signals for the resistors $R_{r e f}$ and $R_{x}$.

when the resistor $R_{x}$ is measured and $R_{B I A S}>R_{x}+R_{\text {ref }}$, the time constant of this $\mathrm{RC}$ circuit is

$$
\tau_{i}=\left(R_{x} / /\left(R_{r e f}+R_{B I A S}\right)\right)\left(C_{s}+C_{p}\right)
$$

where $C_{s}$ represents the sampling capacitor, $C_{p}$ is the parasitic capacitor due to, for instance, a long cable wire connecting $R_{x}$ to the interface. This time constant results in a relative error for the result

$$
\varepsilon_{N L}=\exp \left(-\frac{T_{p}}{\tau_{i}}\right)
$$

where $T_{p}$ is the time period of the output signal of the oscillator.

Example: When the parasitic capacitance is $2000 \mathrm{pF}, C_{S}=$ $28 \mathrm{pF}, R_{B I A S}=2.2 \mathrm{k} \Omega, R_{\text {ref }}=R_{x}=100 \Omega$ and $T_{p}=2 \mu \mathrm{s}$, then, the time constant is about $194 \mathrm{~ns}$. This time constant will result in a nonlinearity of about $33 \mathrm{ppm}$.

\section{B. Switch Clock Feedthrough}

Because the sampling switch $S_{s}$ consists of two switches that are controlled by opposing clock signals, the effect of switch clock feedthrough can partly be canceled. Moreover, the use of CMOS switches will diminish the effect of switch clock feedthrough because these require opposing clock signals.

\section{Noise}

Noise in the resistance measurement with the interface shown in Fig. 1 originates mainly from two parts: the oscillator and the period measurement with using the microcontroller. These two noises can be expressed by [7]

$$
\begin{aligned}
\varepsilon_{n o} & =\sqrt{\frac{C_{p i n}}{C_{s}^{2}} \frac{2 f_{T} C_{i n t} v_{e q}^{2}}{V_{c c}^{2} N}} \\
\varepsilon_{n q t} & =\frac{1}{\sqrt{3}} \frac{1}{N T_{p} f_{c}}
\end{aligned}
$$

where $f_{T}$ is the bandwidth of OTA, $v_{e q}$ the equivalent input noise $(\mathrm{V} / \sqrt{\mathrm{Hz}})$ of OTA, $C_{\text {pin }}$ the parasitic capacitance at input of OTA, $f_{c}$ the sampling frequency of the microcontroller, and $N$ the measured period number.

\section{MEASUREMENT Results}

The novel method has been tested using the circuit shown in Fig. 1. The interface is implemented with an ASIC, which

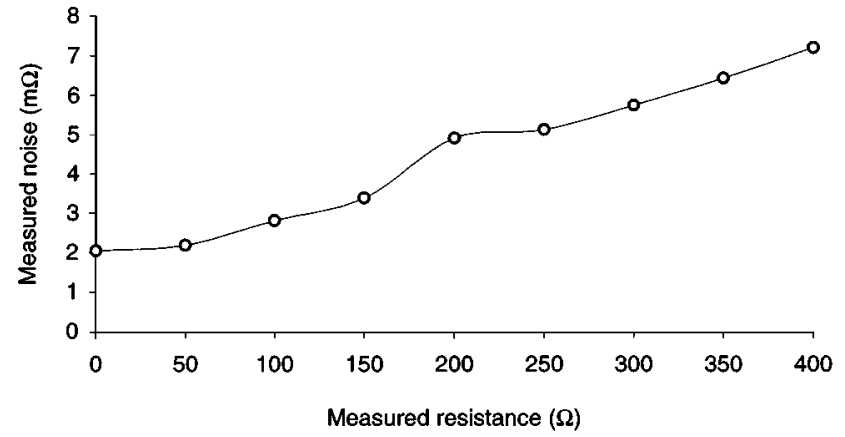

Fig. 3. Measured noise of the interface.

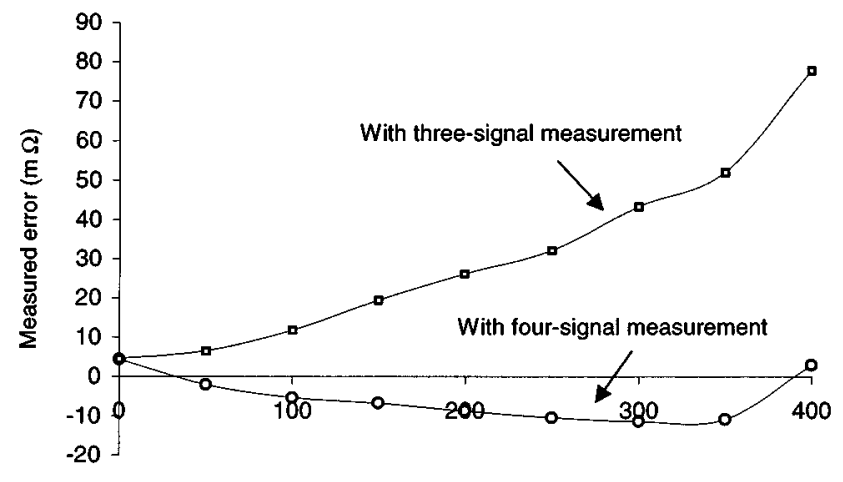

Measured resistance $(\Omega)$

Fig. 4. Measured absolute error of the interface.

was presented in ref. [4]. This ASIC has been realized using low-cost CMOS technology. A microcontroller of the type INTEL D87C51AF, which has a $3 \mathrm{MHz}$ counting frequency, is employed to measure the output period of the interface, to process the data and to communicate with the outside digital world. The system is powered by a single $5 \mathrm{~V}$ supply voltage.

The resolution and the accuracy of the interface have been tested for the case that $R_{\text {ref }}=100 \Omega$ and $R_{x}=0 \Omega$ to 400 $\Omega$ with a measurement time of about $100 \mathrm{~ms}$. The measured resolution amounts to $7 \mathrm{~m} \Omega$ (Fig. 3). Fig. 4 shows the measured error of the interface with the use of the three-signal method and the new method.

It is shown that the interface can measure the resistor of 0 to $400 \Omega$ with an accuracy of $11 \mathrm{~m} \Omega$ using the new multiplesignal calibration technique. Compared to the measurement results based on the three-signal technique, the accuracy of the interface has been significantly improved.

\section{CONCLUSION}

In this paper, it is shown that the accuracy of sensor interfaces with auto-calibration can be considerably improved by performing an extra offset measurement. Applying this technique, a low-cost interface with a high accuracy and good long-term stability has been realized. Experimental results show that the interface can measure the resistor with a resolution of $7 \mathrm{~m} \Omega$ and an accuracy of $11 \mathrm{~m} \Omega$ in a measurement time of about 100 $\mathrm{ms}$. The proposed resistive-sensor interface is very suitable for implementation in low-cost CMOS technology. 


\section{REFERENCES}

[1] G. C. M. Meijer and A. W. van Herwaarden, Thermal Sensors. Bristol, U.K. and Philadelphia, PA: IOP, 1994.

[2] Industrial Platinum Resistance Thermometer Sensors, Amendment 2, IEC-751, 1995-07.

[3] F. M. L. van der Goes and G. C. M. Meijer, "A universal transducer interface for capacitive and resistive sensor elements," Analog Integrated Circuits Signal Process., pp. 249-260, Nov. 14, 1997.

[4] F. van der Goes, "Low-cost smart sensor interfacing," Ph.D. dissertation, Delft Univ. Technol., Delft, The Netherlands, Apr. 1996.

[5] G. C. M. Meijer, J. van Drecht, P. C. de Jong, and H. Neuteboom, "New concepts for smart signal processors and their application to PSD displacement transducers," Sens. Actuators A, vol. 35, pp. 23-30, 1992.

[6] X. Li, G. W. de Jong, G. C. M. Meijer, F. N. Toth, and F. M. L. van der Goes, "A low-cost CMOS interface for capacitive sensors and its application in a capacitive angular encoder" (in Special issue on smart sensor interfaces), Analog Integr. Circuits Signal Process., vol. 14, no. 3, pp. 221-231, 1997.

[7] F. N. Toth, G. C. M. Meijer, and H. M. M. Kerkvliet, "A very accurate measurement system for multielectrode capacitive sensors," IEEE Trans. Instrum. Meas., vol. 45, pp. 531-535, Apr. 1996.
Xiujun Li was born in Tianjin, China, on February 19, 1963. He received the B.Sc. degree in physics and the M.Sc. degree in electrical engineering from Nankai University, Tianjin, in 1983 and 1986, respectively. In 1997, he received the Ph.D. degree from the Department of Electrical Engineering, Delft University of Technology, The Netherlands.

In 1996, he was an Assistant Researcher at the Faculty of Information Technology and Systems, Delft University of Technology, where he was involved in research and the development of smart capacitive sensors and low-cost, highperformance interfaces for smart sensors. Since May 1997, he has worked part time for SMARTEC B.V. on the smart temperature sensor and smart sensor interface. His research intrests include smart sensors, smart sensor systems, and smart signal processing.

Gerard C. M. Meijer (SM'99) was born in Wateringen, The Netherlands, on June 28,1945 . He received the ingenieurs (M.S.) and Ph.D. degrees in electrical engineering from the Delft University of Technology, Delft, The Netherlands, in 1972 and 1982, respectively.

Since 1972, he has been part of the Laboratory of Electronics, Delft University of Technology, where he is an Associate Professor, engaged in research and teaching on smart sensor systems and analog electronics. In 1984 and part-time during 1985-1987, he was seconded to the Delft Instruments Company, Delft, where he was involved in the development of industrial level gauges and temperature transducers. In 1996, he was one of the founders of the company SensArt, where he is a consultant in the field of sensor systems.

Dr. Meijer is a member of the Netherlands Society for Radio and Electronics. 